Pusceddu, C. and Blumberg, G. (2017) 'The use of Building Technology to support Disaster Resilience: The case study of Air Shelter House', International Journal of Disaster Resilience in the Built Environment, 8 (2)

DOI: https://doi.org/10.1108/IJDRBE-11-2015-0057

This document is the authors' Accepted Manuscript.

License: https://creativecommons.org/licenses/by-nc-nd/4.0

Available from RADAR: https://radar.brookes.ac.uk/radar/items/4983bd54-0f10-4fd1-9fae-838241ad41a7/1/

Copyright $(\subseteq$ and Moral Rights are retained by the author(s) and/ or other copyright owners unless otherwise waved in a license stated or linked to above. A copy can be downloaded for personal non-commercial research or study, without prior permission or charge. This item cannot be reproduced or quoted extensively from without first obtaining permission in writing from the copyright holder(s). The content must not be changed in any way or sold commercially in any format or medium without the formal permission of the copyright holders. 


\title{
The use of Building Technology to support Disaster Resilience: \\ The case study of Air Shelter House.
}

\begin{abstract}
This paper reports on a study to investigate the feasibility of Thermal Reflective Multi-layer System (TRMS) as support for Disaster Resilience. It is an innovative insulation system, developed from space engineering studies, lightweight and is characterized by a thermal conductivity of $0.038 \mathrm{~W} / \mathrm{mK}$, making it a strong candidate for inexpensive shelter after disaster design.

One of the results of this study is a proposal for the Air Shelter House (ASH), a new concept design of a shelter based on TRMS. The combined use of TRMS with low cost building materials and a 3D printer system for the construction joints provides a good comprise of building cost and energy efficiency performance. Such an innovative design supports disaster resilience during response, reconstruction and mitigation phases and is suitable for a wide variety of cultural and environmental situations where energy efficiency is important.
\end{abstract}




\section{Introduction}

It is estimated that in 2014 approximately 30 million people worldwide were living in the open or in makeshift shelters. The surge in homelessness is the result of both man-made and natural disasters. The response from the international care community is to propose a number of designs for temporary shelters. Design decision are based on the traditional engineering compromise between, cost, effectiveness, energy conservation, ease of transport and assembly.

The UN's Hyogo Framework for Action (UNISDR, 2005; ISDR, 2007), addressed the requirement to adopt building technology as a fundamental element in augmenting disaster resilience. One of the outcomes of the framework is to encourage preventative community action to reduce the impact of disaster. In addition to made-made disasters, notably war and other social upheaval, there is the possibility that there will be an increase in destructive storms and extreme weather events due to the disruptive affects of global warming and the consequent higher intensity of the storms, as well as other damaging impacts such as ocean acidification and rice-yield-impairing heat (Allan \& Soden, 2008). Also to be expected is an increase in the impact of natural events (NOAA 2009; Arndt et al., 2010) on human settlements. The number of occurrences has increased 30\% to 35\% from 2005 .

The occurrence of extreme events presents a real threat to the built environment, an economically and structurally important sector.

From this perspective, and considering the large numbers of occurrences in developing countries, emergency reconstruction could be an opportunity to introduce energy efficiency and sustainable practice, in parallel with the practice to preserve local building culture (Shelter Centre et al., 2011). For this reason, it is fundamental that any new technology introduced is in agreement with local building process, materials and social needs. These are considered essential considering that many members of the community would be traumatized after a disaster.

One way to encourage local involvement in the reconstruction process is to involve the community and other stakeholders early on in the reconstruction activities, starting with the design phase (Shelter Centre et al., 2010; Shelter Centre et al., 2011). New technology, if used, should be easily adapted to local use with inhabitants learning to manage and maintain the buildings themselves. The community and local 
stakeholders could be considered as practitioners connected with research trying to achieve the best solution that would guarantee the ability to return back to normal conditions.

This process is compatible with local cultural, environmental, sociological requirements and the opportunity to introduce energy efficiency, supporting the right to adequate housing (UN-HABITAT, 2009, UNHCR, 2014) and economic growth.

\section{Methodology}

In view of disaster resilience and management studies, this research considers innovative technology to obtain a balance between economic, environmental and sociological aspects. The aim of the study is to demonstrate the possibility to use building technology to achieve the best solution in this contest and optimising the usability and energy efficiency. The methodology follows four different task:

- Task 1: The state of the art of shelter. This review will summarize the present global state of art of shelters, shelters features and response-phase requirements.

- Task 2: The study of building technology systems will identify the potential application of TRMS. These will be conducted in the laboratory as well.

- Task 3: Feasibility study. This task will define the concept of the Air Shelter House (ASH) in addition to an evaluation of the building prototype, the Air Shelter House Pillow (ASH Pillow), through simulations with TRNSYS and THERM simulation environment.

- Task 4: Studies on the application of ASH. These studies will investigate the uses of the ASH Pillow in the reconstruction, design of new buildings, and analysis by the THERM software.

The state of the art of shelters is divided into three different categories shelter classification (IFCR, 2013), and physical and functional characteristics of the housing systems. The first to consider is the Emergency Tent (ET). After a disaster and during recovery, ET provides easy transport, assembly and the possibility to be reused at other sites. The second group is the more substantial Shelter (SH) category. Studies have been performed to measure the proportion between building envelope flexibility, weight and thermal performance that would ensure internal comfort and energy efficiency. The last group is the more permanent Housing (H). There are numerous examples of best practice from 1995 to 2013 and considerable progress has been 
made in the application of certain technologies, in particular, the T-shelter, Progressive and Core Shelter and other long-term solutions.

Task 2 considers the investigation of TRMS. This is an innovative housing technology originally developed for the aerospace industry. It is based on a reflective films ( $\mathrm{Li}$ and Cheng, 2006), which has superior thermal performance while still being comfortable to wear. TRMS is composed of different types of films and is produced by combining TRM and other materials separated by air gaps. In this case study, the TRM contains 19 layers (ACTIS SA, 2007), which alternate between aluminium foil and wadding, foam layers, films all sewn together to form a thin insulating blanket.

This configuration was chosen because it provides high insulating performance with a thickness three to four times thinner than traditional materials (including air spaces), complying to the international certification Institute (BM-TRADA, 2014). This technology takes advantage of all three modes of heat transmission (radiation, conduction and convection) and reflects heat outside the envelope during summer season, while retaining it inside the building during the winter months. The TRMS is waterproof its thermal performance reduces internal condensation (RIMA_I, 2002; Seriacaroupin et al., 2007).

Numerous studies from international laboratories have been conducted to evaluate thermal performance if TRMS using different methodologies and conditions (Tenpierik \& Hasselaar, 2012; Salvalai et al., 2015).

Furthermore, the TRM and TRMS application in Civil Architecture are examined to consider the possibility of use of this insulation inside domestic dwelling. Seven different case studies are considered: four from Australia and three from Italy. This wide-spread, in view of different possible scenario in terms of climate, internal functions and intervention typology.

Finally, laboratory experiment are reported from research studies that use a guarded hot plate apparatus. These were done in collaboration with the Measurement Laboratory of Politecnico di Milano, Campus of Lecco. Two different test samples were used, the first was made by TRM and second was made by TRMS. This made it possible to investigate improvements associated with air layer created with a polyester membrane (Salvalai et al., 2015). The results of the test procedure showed general agreement with the method and the standard ISO 6946:2007 in which thermal resistance and thermal transmittance are calculated. Temperature was set at three different values, two for the TRM and one for the TRMS 
(Imperadori et al., 2013). Thermal parameters are not directly measured, for this reason, the uncertainties of error propagation were calculated (Salvalai et al., 2015).

Between the completion of the second and the third task, the Air Shelter House (ASH) proposal was made to implement a housing technology based on the use of TRMS.

For the third task, an economic and technical comparison is made between this innovative solution and the current solution that is commonly used in the response and reconstruction phases of disaster recovery.

A building component, based in TRMS technology, is investigated to understand feasibility of ASH in different solutions. It is a system made by modular components, called ASH Pillow, which is a thermal, flexible skin to use as internal or external envelope for the reconstruction of damaged buildings, for new buildings, and micro-architecture or tent, according to the local construction technical and materials (Imperadori et al., 2014).

In the fourth and final phase, the application of ASH is considered in reconstruction and building design. During this application it will be possible understand potential of material application to different structure, in support to the design process, and considering the prototype study of the two new shelters design.

\section{The Air Shelter House Case Study}

Bologna \& Terpolli (2005) and Comerio (2013) confirm that post disaster intervention requires an original approach. For this reason, it is difficult to compare disaster loss and recovery program in different countries when local conditions make each situation unique. However, some generalizations can be made (Comerio, 2013), and analysis of experience can help us to understand which may be the general element, common to all countries and to within all disaster management phases. In agreement with these investigations, a building component to adapt to general framework was performed based on ASH concept. These are shown in Figure 1. 

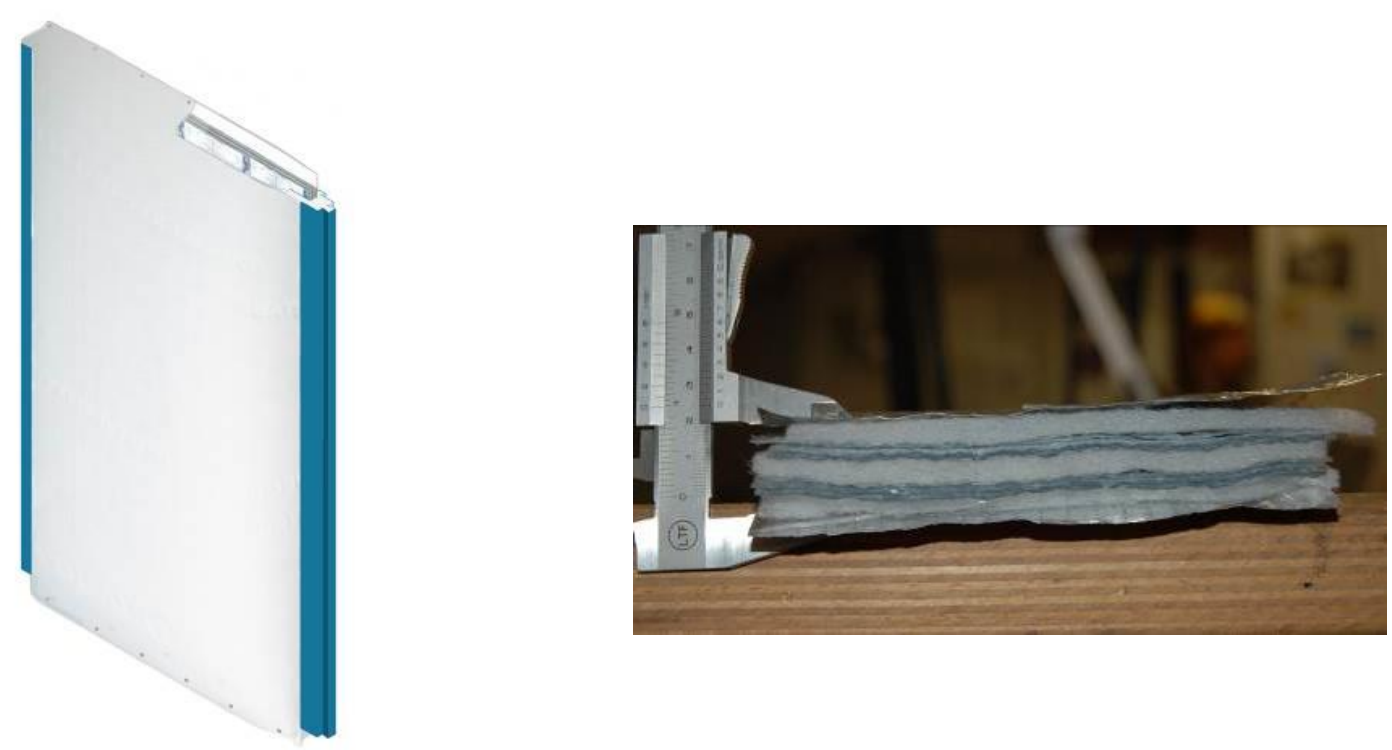

Figure 1-2: Air Shelter House Pillow design; TRM insulation material. Credits: Author.

The ASH Pillow could potentially make the application of TRM (Figure 2) easier and from different users without the support of specialist tools. For example, during the reconstruction of building in Iran after the 1997 earthquake (Ghafory-Ashtiany, 1999), the importance of good construction process management after disaster was demonstrated. In this example, the management was primarily concentrated with providing the construction materials and budget. The regional engineers did not play a role in the reconstruction and this was later realised to be a poor practice as there was evidence of a poor quality construction.

The main advantage of ASH Pillows is that it can be used for building joints that can easily be applied by unskilled workers, without the normal problems associated with construction of a building envelope. ASH Pillows have the added advantage of flexibility of use as they are able to adapt to different kinds of structures as being suitable for use as tents in refugees camps, or as T-shelter or Core/Progressive shelters. TRM has a lifespan of 10 years (ACTIS SA, 2007), which approaches the durability required for permanent house.

The materials selected to create insulation panel were limited to those available from the open market. These were low cost, weather resistant and lightweight and with good thermal performance. In the first research step, the plastic joints were made with vulcanized rubber. As there was no opportunity to recycle the rubber and the design was direct to the use of materials, as ABS or TPE, then it can be produced by a 3D printer system, while still maintaining a thermal conductivity of $0.18 \mathrm{~W} / \mathrm{mK}$. This solution was conducted in parallel with the recent projects of ONG and Onlus, where 3D printers are designed with recycled elements to be available in developing countries as well (Sardegna Ricerche, 2014). In this way, the producing of 
building components can be performed by builders with basic skills, with the intent to reduce cost and to give new impulse to local economy after disasters.

\section{Results And Discussion}

Considering that $31 \%$ of funds allocated for reconstruction phase in 2014 were invested in housing construction (The World Bank, 2014) it would be reasonable to conserve financial resources for this in combination with energy consumption in order to reduce global warming. This solution must be done in a socially sympathetic manner by providing better domestic comfort for users and also contributing towards reducing energy wastage. In this way, it will possible to reduce vulnerability of housing systems too, reducing economic losses, and cost for housing reconstruction, according with HFA (UNSDR, 2005), Sendai Framework for Disaster Risk Reduction (UNSDR, 2015).

The use of state of the art emergency tents, T-shelters and Core/Progressive shelters underline the direct relation between the cost and the improvement of the effectiveness of the housing system. Moreover, there is negative correlation between the thermal performance of a system and its flexibility, in terms of weight and ease of assembly.

The ten ET systems, in particular, are designed considering the requirements of easy transport, assembly and the possibility of reuse in other situation, as required during disaster and response phases. However, the particular nature of ET as it is used for refugees, increases the possibility that these solutions are used for periods longer than 12 months.

The state of the art reveals a scenario where technology improvements are made to increase durability of these housing systems UNHCR (2006), UNHCR (2010) and IFCR (2011). These reports are examples of this, as described in the ET specifications of "Family tent" and "Light-weight tent". However, technological improvements are often too expensive considering limited availability of funds in this sensible phase, and the use of innovative solutions could be limited from the price. Nevertheless, hybrid system of ET and TShelter demonstrates the interest of the companies to find a balance between internal comfort and flexibility, with example of system that have $15 \mathrm{~m}^{2} \mathrm{~K} / \mathrm{W}$ of thermal envelope performance, but with a basic design that could be improved. 
The SH case studies demonstrate that it is possible to observe the negative correlation between envelope flexibility, lightweight of the system, and thermal performance, but that these ensure internal comfort and energy efficiency.

The sixteen case studies chosen were done between 1992 to 2013 . These provide a good overview of the technology used during these years and include a number of systems with different final application, such as housing or service units. The result of these cases, show that many of these solutions are very innovative. For example, by using recycled envelope to optimize system cost and to reduce $\mathrm{CO}_{2}$ consumption. These are done within the limits of (1) small size prototype studies, (2) limitation of a low R-value, (3) architectural integration inside the local culture. Some of these solutions are less practical as the cost of temporary shelter are higher than full building reconstruction (Shelter Centre et al., 2011).

One item of note is that in the SH case studies, the during the application of the flexible system analysed in the $\mathrm{H}$ cases an attempt was made to change the system during the reuse, with the introduction of a ductile envelope able to adapt to a different architectural design.

The price of these shelters has to be considered in comparison with previous construction methods. Unfortunately, reuse sometimes involves some additional cost. The case of Duzce, in Turkey, on 1999, is an example of these issues. In fact, the cost to move $200 \mathrm{~m}^{2}$ (8 units) to a nearby community to use as a Sports Centre was US\$8000, which included transport, installation of electricity, water, and a new roof, around $40 \%$ more of initial building cost (Johnson, 2007).

These results indicate the advantages to be focus on the study and application of technologies for buildings envelope, contrarily of the design of a total shelter system. If we focus on the envelope, it will be possible rebuild using the local typologies structure, in agreement with the environment, the local culture and with the change to increase the resident economy. Besides, in view of a possible reuse, transport and insertion phases can achieve lower costs considering the characteristics of easy application and transfer of a single component than a full system. Furthermore, the use of a building technology can be used to adapt it to different structures. In the case of ASH Pillow, this could allow neglecting the important connection between population and attachment to a place and providing a balance between technology and cost.

The high thermal performance of TRMS can guarantee internal comfort for users as well as energy efficiency. To ensure this, a comparison is made between a general ET (Case1), and ET with TRMS made of 
polyester as ASH (Case 2). (Salvalai et al., 2015). This was done by performing a software simulation with TRNSYS. Case 2 concerns a material with a higher thermal value, which is of benefit principally during the night when the presence of people inside the tent and when heat is reflected inside the housing system.

The result of the simulation indicate that energy consumption of approximately $1225 \mathrm{kWh}$ is required for the general case study, and $388 \mathrm{kWh}$ for Case 2. According to the temperature analysis, the envelope with TRMS shows the best control, and it is possible to assert that there is high energy savings when the thermal performances of the envelope are guarantee thanks the technology insert, with a heating loads reduction sets up to $69 \%$ (Salvalai et al., 2015).

The increase in thermal performance can be attributed to both TRM and TRMS as it is also demonstrated from several case studies done by analysing from different countries and supported from international and local thermal performance certification.

The four Australian case studies represent the application of TRM in four different projects with either one or two floors. The TRM insulation material is located in the wall cavity and under the roof. The structure is made up of different materials (primarily steel and wood) and has either a load bearing wall system or punctual bearing structure. The BCA national certification and the National Awards document the value of using this insulation. High overall building performance is guaranteed independently by a large surface area, load bearing structure or a TRM positioned within the envelopes. As shown from the different case studies, the use of this innovative material help to optimize the result of a good project design, also has sustainable criteria.

All projects are pilot design adopted to varied Australian cities and increase energy buildings efficiency. These projects are still recognized as a best practice to follow.

The Italian case studies, moreover, represent three possible various scenario for TRMS applications.

In particular, the Case A presented is a building located at Monte Rosa. It is a modular housing system used as a steel-structure bivouac using TRMS inside the shell envelope.

Case B is located in a seismic area, close to Aquila, Abruzzi and is being used as a commercial office building. In this case, TRMS is used in the envelope (roof, walls, floor) with the superstructure made from X-Lam load bearing structure. 
Case $\mathrm{C}$ is a townhouse located in an historical Sardinian city center where heritage characteristic require preservation of existing frontages. It is used as private housing. In this case, TRMS is used in the envelope as internal thermal skin and the load-bearing walls are made of stone.

In any case, the use of TRMS helps to achieve a practical solution that allows for low energy consumption and also protects against the climate, geological risks and architectural requirements. The limited thickness of TRMS is helpful to achieve space savings, required in this limited area and the contemporary presence in the walls, roof and floor in the third case study help the building to attain an $\mathrm{A}+$ Classe $\left(6,71 \mathrm{KWh} / \mathrm{m}^{2}\right.$ year energy consumption). This is the highest certification for energy efficiency obtainable for Italian buildings.

The high thermal performance of TRMS are reported in the literature as well. Various studies of laboratory experiments of TRM and TRMS are described here. The data concern tests of $25 \mathrm{~mm}$ thick TRM.

The only exception, in the literature scenario, is represented from In situ tests, where is recorded a higher value compare to average results. The insulation material, in fact, is designed to be used in direct contact with the outside environment. In this context, the irradiation characteristics can achieve the best performance because of direct interaction with climate and environmental conditions. These characteristics are not normally replicable in the laboratory. The measured conductivity in literature varies from 0.03 to 0.05 $\mathrm{W} / \mathrm{mK}$ with an average value of $0.037 \mathrm{~W} / \mathrm{mK}$.

The choice to perform the Guarded Hot Place Apparatus experiment with two different tester, Tester 1 made by TRM and Tester 2 made by TRMS (Salvalai et al., 2015), allowed to investigate concerning possible improvement connected with air layer; in this case created with a polyester membrane. Despite the limited thickness of air gaps $(1 \mathrm{~cm})$ a rising of thermal performances could be achieved. The conductivity, in fact, are around $0.033 / 0.043 \mathrm{~W} / \mathrm{mK}$. Better studies can consider possible increasing of air gap thickness, about 2 $\mathrm{cm} / 25 \mathrm{~cm}$, in agreement with the tests already carried out in the University of Reunion (Seriacaroupin et al., 2007), to improve thermal performance of the TRM, air and polyester system.

Considering these, a building component was projected based on TRMS as the ASH concept, named the ASH Pillow, where air cavity is $2 \mathrm{~cm}$.

With a transmittance of $0.81 \mathrm{~W} / \mathrm{m}^{2} \mathrm{~K}$ this insulation system could be used as a thermal skin for buildings ensuring internal comfort for users. Considering software simulation with THERM, thermal performance is satisfactory, however, there are some shortcomings. It is possible observe thermal losses in the corner 
conjunction between building panels. These can easily be reduce with the application of radiant barrier, also made of TRM. This is a common practice already used in the civil buildings structure.

Lightweight (less than $1 \mathrm{~kg}$ for each panel) and standard dimension comply with ISO6346 (Containers code: size and type for intermodal shipping containers), assent a practical transport of the elements inside shipping container, often use in these phase by rescuers. Packages could be made by one person, in five simple steps in order to move the insulation skin from different shelter solution without the support of professional tools. Simplicity of ASH Pillow allows involving locals in the construction phases during all the response and recovery process, and an easier maintenance of the housing system in the future.

Case studies A1 (Nakagin Tower reconstruction), Case A2 and Case A3 (Scaffold House and Cardboard House new construction), demonstrate feasibility of ASH Pillow to be safe during earthquake, adaptability of the building component to different structure-context, and high thermal performance in agreement with functional and internal comfort.

Case A1 concerns the elaboration of data of in Japan and the study of Nakagin Tower structure. This was done with the collaboration of Japanese professionals.

The conjunction between probable deconstruction decision of Nakagin Tower (Figure 3) and Tohoku earthquake encourage consideration about reconstruction, but in the same time, to preserving heritage. Reference to the traditional buildings, such as the "Tea house", by Toshiko Suzuki, and high thermal performances of “Ape Tau”, by Atelier2.

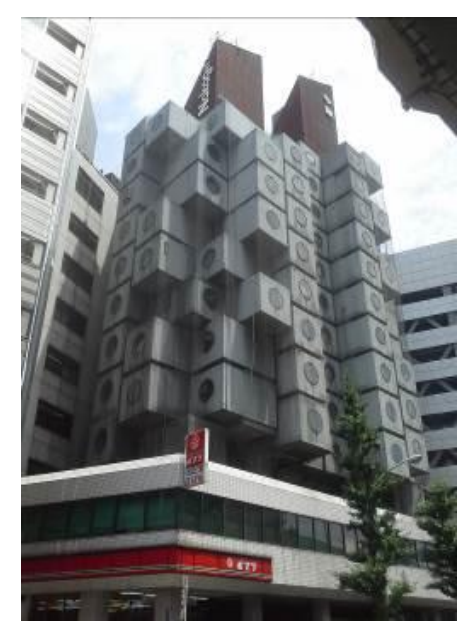

Figure 3: Nakagin Tower, Tokyo, Japan. Credits: Author. 
According to disaster reconstruction targets, the design is based on the original steel structure with a new building envelope installed. This is done in consideration of asbestos removal, done without changing architectural features, connected to Metabolism architectural movement, and housing system memories.

The envelope is made from two different skins: the external one in sandwich panels, and the internal one, in ASH Pillow (Figure 4). The outside shell is made of Polyurethane wall panels with concealed fastening and sheet roofing panels of $0.08 \mathrm{~m}$ thick, with a transmittance respectively of 0.25 and $0.23 \mathrm{~W} / \mathrm{m}^{2} \mathrm{~K}$ and a weight of $14,93 \mathrm{Kg} / \mathrm{m}^{2}$ (Marcegaglia, 2013). The ASH Pillow has a weight around $0.70 \mathrm{~kg} / \mathrm{m}^{2}$ and a thermal conductivity around $0.81 \mathrm{~W} / \mathrm{m}^{2} \mathrm{~K}$, in the section where there is the TRMS.

The study of internal temperature surface is made to understand shell performance, compared with current situation. The most disadvantageous configuration are considered during finite elements simulation, with the exposition of floor to external temperature. To offset floor thermal performance a layer of TRM type ACTIS Triso Super 10 was added in the installation cavity below the floor (Figure 5).
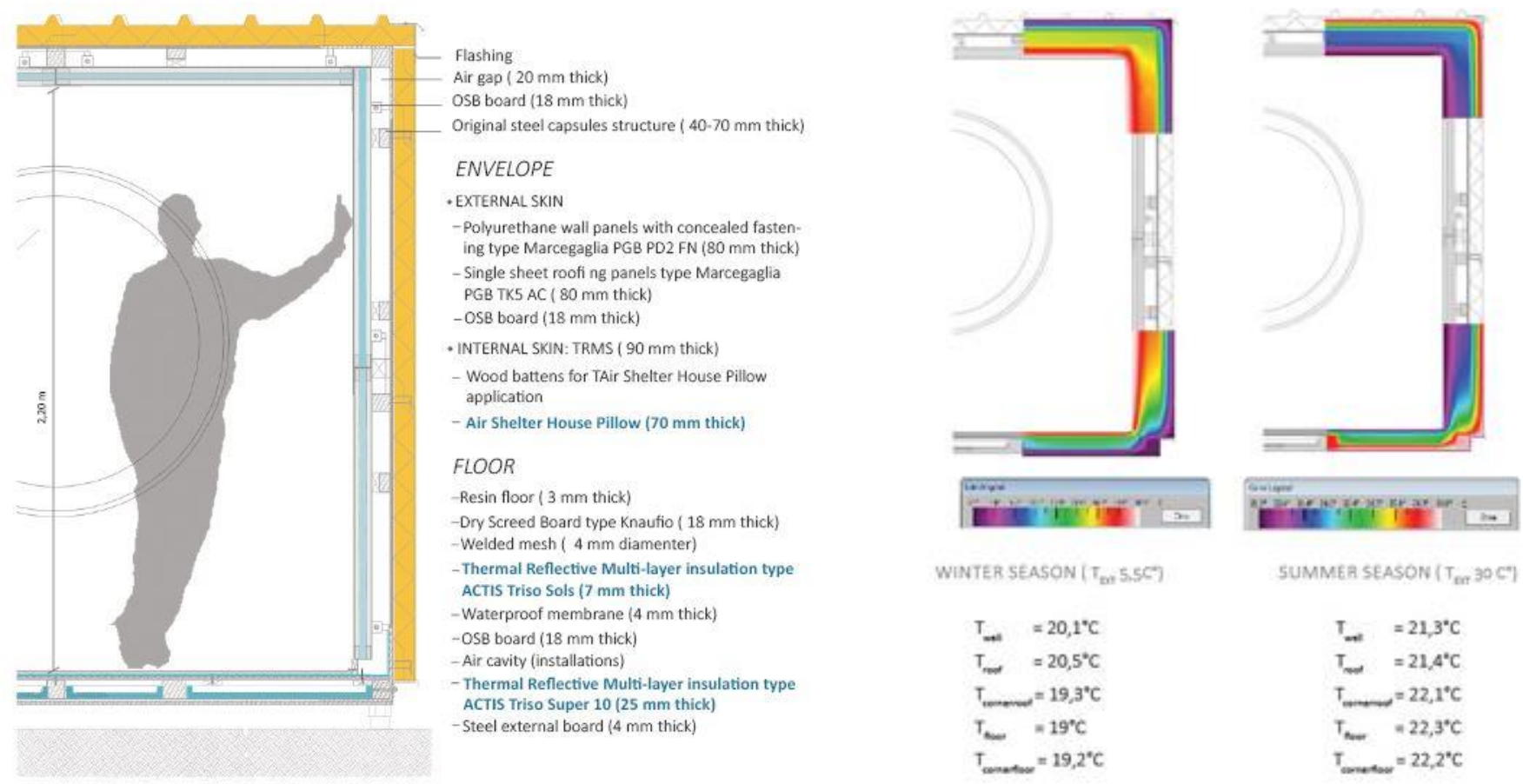

Figure 4-5 Therm of winter and summer season of insulated capsule design with ASH Pillow. Credits: Author.

The results shown a considerable improvement of internal comfort with an increasing of surface temperature of $8^{\circ} \mathrm{C}$ in the wall, around $11^{\circ}$ in the floor during winter season. In addition, there is a decreasing of surface temperature of $6^{\circ} \mathrm{C}$ in the floor during summer season.

ASH Pillow have an emissivity of 0.1 , according to possible interaction between the reflective layers and internal thermal loads, increasing internal temperature during the winter. 
Thanks to the flexibility of ASH Pillow, in combination with Sandwich panel, in the end, it will be possible to preserve the original structure and added an internal layer without compromise internal function.

$13 \%$ of internal space could be earned, creating a housing space available for single people or generating a small community with possible capsule composition as show from the project "Metabolizing Metabolism" (Pusceddu et al., 2013).

Case A2 is the Scaffold House, a housing unit projected with materials already present in the market, but used in an innovative way. The design comes from a final project of Politecnico di Milano students, Azzolini et al. (2012), afterwards it has been most thoroughly investigated in collaboration with companies, though also workshop experience (Figure 6).

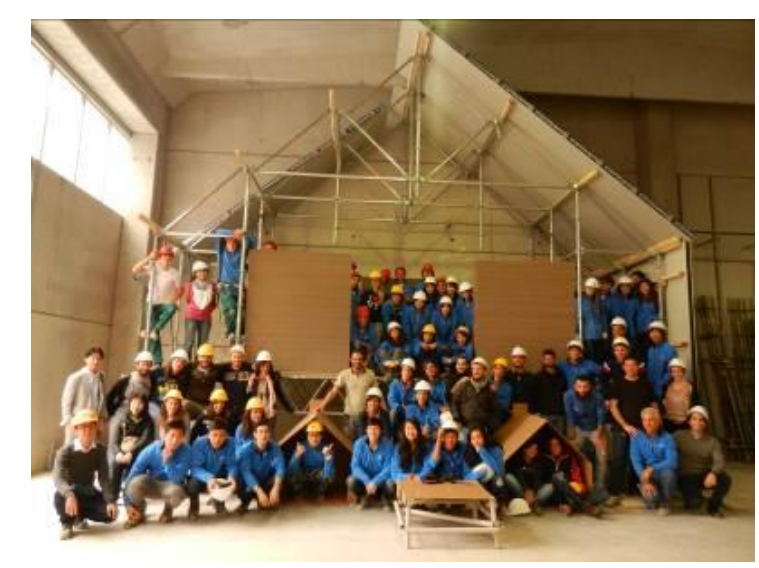

Figure 6: Workshop, Politecnico di Milano, Lecco, Italy. Credits: Author.

The housing system developed inside the research, after prototype study and integration of new envelope, is an easy assembly modules composed of practical components, as scaffold, sandwich panels and ASH Pillow.

Considering possible internal functions, the envelope is studied to guarantee thermal performance and comfort for users, achieving energy saving according with low consumption and a sustainable development. During the prototype construction, a sandwich panel envelope was experienced, as a faster and flexible new solution.

The envelope shell has same characteristic of Case A1 and combination of two skins can ensure a high thermal performance in a more practice way, compared with original solution (Azzolini et al. 2012). Easy connection and lightweight of ASH Pillow can be in agreement with the timing recorded during the workshop, and with the features to be assembly without specific tools. Flexibility of the panel and joints allows adapting the system to the already design structure, optimizing the building process, with seven 
element less than original version, in agreement with the emergency needs of a fast and easy assembly (Figure 7).
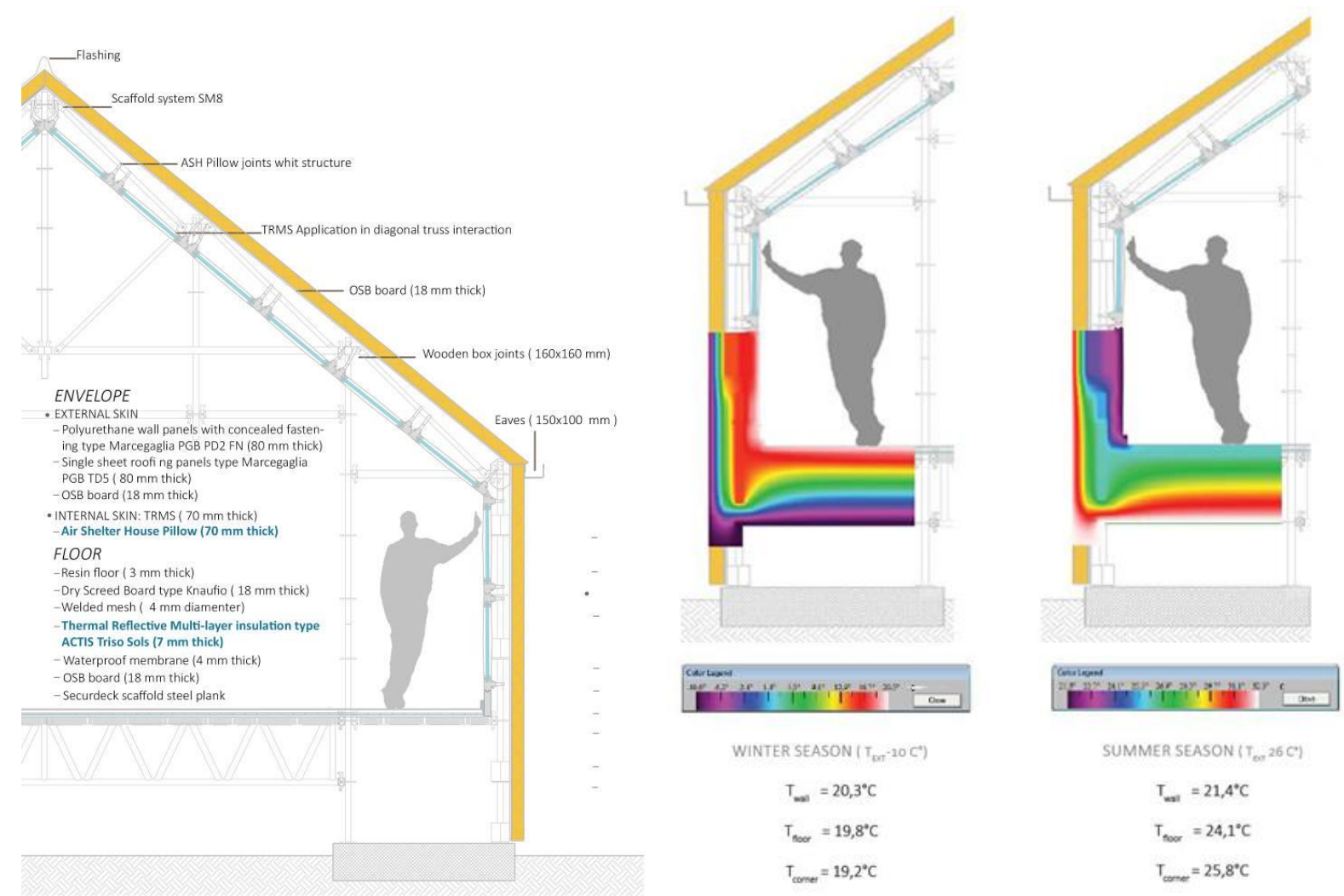

Figure 7-8: Scaffold House research section; Scaffold House color infrared simulation with Therm of winter and summer season of insulated structure with ASH Pillow. Credits: Author.

The internal comfort (Figure 8) is optimized, in agreement with Shelter Centre (2009) specifications, without compromise the original transmittance of the envelope of $0.153 \mathrm{~W} / \mathrm{m}^{2} \mathrm{~K}$. Scaffold House, moreover, could be consider an example where the ASH concept could be a shelter inside a shelter. It should be disassembled at any time, without compromise the all system and reuse in another context, without economic losses. The system made by panels, ensure to tie the envelope to the structure strengthening the all wall, in case of quake (ISDR et al., 2007).

Case A3 is the analysis of ASH Pillow installation in the Paperboard House (Figure 9), an emergency shelter designed by a team of students of Politecnico di Milano for an academic project.

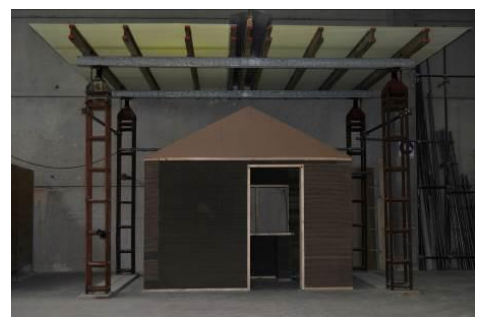

Figure 9: Paperboard House prototype. Credits: Author. 
The work-study is in collaboration with several companies and the project is based on the experience performed on roof system designed in occasion of "Architecture for Archaeological Sites" workshop, made on Agrigento on 27 October to 2 November 2013 in collaboration with JIA-Japan Institute of Architecture (Architect National Chamber of Japan) (Domus, 2014).

The application of the AHS Pillow was performed inside the research study, through software simulation with THERM and study of possible TRM application during the Paperboard House Workshop in Lecco (Figure 10), 4 October 2014, analysing the possibility to optimize thermal performance of the shelter, and internal comfort, without compromise the internal space. In fact, a traditional rigid insulation would produce several conditions in terms of global rigidity of the structure and vibration's transmission: the lightness of the system of less than $700 \mathrm{~g}$ for each panel, make the system adapt to be move in combination with light structure as the cardboard roof, assuring a good behaviour also in earthquake situations. With a lifespan of 10 years (ACTIS SA, 2007), TRM could be also disassembled and reused as in the previous case. This feature is possible thanks to simple joints of ASH Pillow. Lightweight of the system allows locating and moving the insulation layer without specific tools, according with maintenance needs.

Internal constant temperature of $21^{\circ} \mathrm{C}$ confirm possible internal comfort for users, and reduction of heat transfer through the envelope (see Figure 11).

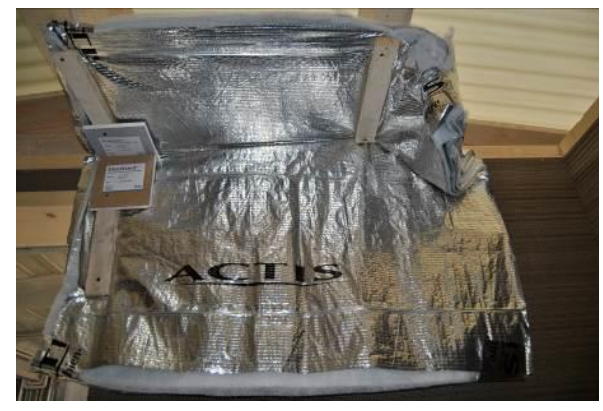

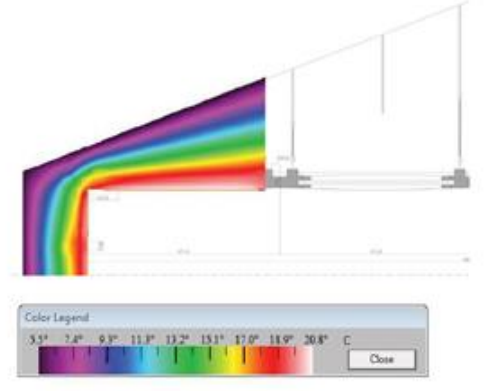

ASH P ROOF INTERNAL TEMPERATURE

$T_{\text {wall }}=20^{\circ} \mathrm{C}$

$\mathrm{T}_{\text {root }}=20,6^{\circ} \mathrm{C}$

$\mathrm{T}_{\text {corner }}=18,1^{\circ} \mathrm{C}$

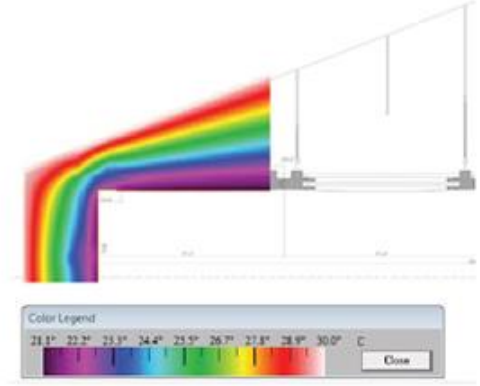

ASH P ROOF INTERNAL TEMPERATURE

$T_{\text {wall }}=20^{\circ} \mathrm{C}$

$T_{\text {roof }}=21,2^{\circ} \mathrm{C}$

$\mathrm{T}_{\text {corner }}=22,5^{\circ} \mathrm{C}$

Figure 10-11: TRM roof-application; Paperboard House color infrared simulation with THERM of winter and summer season of insulated capsule design with ASH Pillow. Credits: Author.

As demonstrated in these case studies, the ASH panel can meet the characteristic of adaptability from a simple shelter to an only one technological element for permanent buildings, as required by Shelter Centre (2009), and Temporary Shelter definition by Corseillis and Vitale, (2005). 


\section{Conclusion}

International community recognized six principal features to achieve a good design for shelter: lightweight, resistance, ductile, practical, modular, according with local culture, and particularly Shelter Centre et al. (2011) assert the importance for shelter to be relocated or reuse in more context.

Notwithstanding, lack of a balance between economic aspects and shelter performance does not allow to provide the best possible approach because of economic disasters and the general weak economic conditions in poor countries.

To be in agreement with international shelter standard and economic aspects, this study focuses on building envelope technology, possibly at the expense of the design of the total shelter system. The Air Shelter House, because of his lightweight and flexibility, make easier to rebuild, when it is possible, respecting the environment and with the change to increase the resident economy. In addition, in view of a possible reuse, transport and insertion phases could be facilitated, with lower costs considering the characteristics of easy application and transfer of a single component than a full system. Victims of trauma could be involved in reconstruction process, helping their path to back to "normality". Moreover, new opportunities for local economy could be created, involving locals in the complete process, and reducing total cost of reconstruction housing projects.

Thermal performance of $0.038 \mathrm{~W} / \mathrm{mK}$ of TRM and modularity of the insulation system ensure to combine the general concept of ASH with different types of structures, and with different kind of materials, guaranteeing the possibility to reconstruct using local building technology construction, materials, and according with local culture (Shelter Centre, UN \& DFID, 2010). High thermal performance, furthermore, ensures internal comfort for users, which needs particular attention considering traumatic experience and possible psychological and physical diseases, caused by the events. Simulation of ASH Pillow as Emergency tent system shown a lowering of energy consumption of $69 \%$ respect to simple standard model (Salvalai et al., 2015), concurring with the aims to decrease Global Warming.

The design of ASH, especially of building component, shown the possibility to use technology in support of response and mitigation, crossing the reconstruction period, reducing vulnerability and supporting disaster resilience. 
In the end, according with the result of State of art, laboratory test and economical behaviour during emergency management, the conclusion of this research is that a building technology, in this case Thermal Reflective Multi-layers System, could be used as important tools, particularly, during response and reconstruction, in order to try to achieve the best result.

Possibility of patent is evaluated to improve study of ASH Pillow, through production of a real panel prototype, to perform more accurate laboratory test, concerning thermal performance and design optimization.

\section{References}

ACTIS SA (2007) “ACTIS TRISO Super 10 Installation Guidelines”, ACTIS SA, Chippenham Wilts, United Kingdom.

Allan, R. P.\& Soden, B. J. (2008) 'Atmospheric warming and the amplification of precipitation extremes’, Journal Science, Vol. 321, pp. 1481-84.

Arndt, DS., Sanchez-Lugo, A, Crouch, J, Heim, RR \& Fenimore, C (2010) 'The Climate of 2009 in Historical Perspective', NOAA National Climatic Data Center, North Carolina, United State.

Azzolini,E, Beretta, E, Cerri, F, Imperadori, M (2012) Unita' D'emergenza Termoriflettenti: progetto di un sistema a base di ponteggi per l'edilizia, Thesis project in Italian, Architectural-Engineering School, Politecnico di Milano, Milan, Italy.

Bolin, RC (1993) Household and Community Recovery After Earthquakes, Boulder, CO: University of Colorado Institute of Behavioral Science.

BM-TRADA Certification (2014) Certificate Number BIPS-0105, BM-TRADA Certification Ltd., Limoux, France.

Bologna, R \& Terpolli, C (2005) Emergenza del Progetto e Progetto dell'Emergenza :Architettura contemporaneità, Federico Motta Editore S. P. A., Milan, Italy.(in Italian)

Bologna, R (2010), "Planning and designing post disaster transitional housing: the case of Abruzzo (IT) earthquake", CIB World Building Congress 2010, Salford (UK), Vol. TG63, pp. 132-144.

Comerio, MC (2013) Housing Recovery in Chile: A Qualitative Midprogram Review, Pacific Earthquake Engineering Research Center Headquarters at the University of California, Los Angeles, United State.

Corseillis, T \& Vitale, A (2005) Transitional settlement, Displaced population, University of Cambridge shelter-project, OXFAM.

Domus (2014), Architecture x Archeology, Domus Digital edition, available at http://www. domusweb. it/en/architecture/2014/01/09/architecture_x_archaeology. Html (accessed 22 October2014). 
Em-DATA (2014), The International Disaster Database, available at http://www. emdat. be/database (accessed 29 May 2014)

Ghafory-Ashtiany, M (1999),"Rescue operation and reconstruction of recent earthquakes in Iran", International Journal of Disaster Prevention and Management, Vol. 8, pp. 5 - 20.

IFCR (2011) Family tent, $16 \mathrm{~m}^{2}$, Head, Shelter and Settlemnte Deparrtment, Geneva, Switzerland.

IFCR (2013) Post disaster shelters: ten design, International Federation of Red Cross and Red Crescent Societies, Geneva, Switzerland.

Imperadori, M, Pusceddu, C \& Salvalai, G (2013), 'Thermal-reflective multilayer insulation system in the emergency architecture: The Air Shelter Skin', proceedings of 29th PLEA Conference, Sustainable Architecture for a Renewable Future, 10-12 September, Munich, Germany.

Imperadori, M, Salvalai, G, Pusceddu, C (2014), "Air Shelter House technology and its application to shelter units: the case of Scaffold House and Cardboard Shelter installations," International Conference on Building Resilience, 09-11 September, Manchester, United Kingdom.

International Organization for Standardization (2007), Building components and building elementsThermal resistance and thermal transmittance-Calculation method, ISO 6946:2007.

International Organization for Standardization (1995), Containers code: size and type for intermodal shipping containers, ISO6346:1995.

ISDR (2007), Hyogo Framework for Action 2005-2015: Building the Resilience of Nations andCommunities to Disasters, United Nations International Strategy for Disaster Reduction, Geneva, Switzerland.

Johnson, C (2007), 'Impacts of prefabricated temporary housing after disasters:1999 earthquakes in Turkey', Journal of Habitat International, Vol. 3, pp. 36-52.

Li, P \& Cheng, H (2006), 'Thermal analysis performance study for multilayer perforated insulation material used in space', Journal of Applied Thermal Engineering, Vol. 26, pp. 2020-2026.

Marcegaglia (2013), Metal Building Envelope, Milano, Italy, pp. 24/54.

NOAA (2009), Global Temperatures Well Above Average, Slightly Above Average for US, National Oceanic and Atmospheric Administration.

Pusceddu, C, Imperadori, M (2013), "Metabolizing Metabolism: Reuse Of Nakagin Tower Elements For A Community In Fukushima. Integrating High Technological Performance With Cultural Heritage And Psychological Needs Of Displaced People”. Proceedings Of Android Residential Doctoral School In Disaster Resilience 2013, 23-24 October Limassol, Cyprus, Vol. 1, pp. 47-55.

RIMA-I (2002), Reflective Insulation, Radiant Barriers and Radiation Control Coatings, Reflective Insulation Manufacturers Association International (RIMA-I), Olathe, Unite State of America. 
Salvalai, G, Imperadori, M, Scaccabarozzi, D, Pusceddu, C (2014), "Thermal performance measurement and application of a multilayer insulator for emergency architecture". Journal of Applied Thermal Engineering, Vol. 82, pp. 110-119.

Sardegna Ricerche (2014), Costruiamo insieme una stampante 3D RepRap, available at http://www. sardegnaricerche. it $/$ index. php xsl $=370 \& s=259847 \& v=2 \& \mathrm{c}=3134 \& n c=1 \& q r=1 \& q p=2 \& v d=2 \& \mathrm{fa}=1 \& \mathrm{t}=3$ (accessed 14 October 2014).

Seriacaroupin , J, Miranville, F, Braga, D \& Duran, M. (2007), 'Experimental evaluation of the thermal performance of a building roof including a multireflective radiant barrier', 5th International Conference on Heat Transfer, Fluid Mechanics and Thermodynamic SJ7, 1-4 July, Sun City.South Africa.

Shelter Centre (2009), Transitional Shelter Guidelines, Part draft Shelter Meeting.

Shelter Centre, UN \& DFID (2010), Shelter after disasters, United Nations.

Shelter Centre, DFID, UKAID, IOM-OIM \& Sida (2011), Transitional Shelter Guidelines, Part draft Shelter Meeting, Geneva, Switzerland.

Tenpierik, MJ \& Hasselaar, E (2012), 'Reflective multi-foil insulations for buildings: A review', Energy Buildings, N. 56, pp. 233-243.

The World Bank (2014), Reconstruction Project, available at http://www. worldbank. org/projects (accessed 14 October 2014).

UN-HABITAT (2009), The right to adequate housing, United Nation, Geneva, Switzerland.

UNHCR (2006), Specifications of Lightweight Tent, United Nations, Version 4.0, United Nations, Geneva, Switzerland.

UNHCR (2010), Most frequently purchased items catalogue, United Nations, Geneva, Switzerland.

UNHCR (2014), Global Strategy for Settlement and Shelter, UNHCR strategy 2014-2018, United Nations, Geneva, Switzerland.

UNISDR (2005), Hyogo Framework for Action 2005-2015: Building the Resilience of Nations and Communities to Disasters, United Nations International Strategy for Disaster Reduction, Geneva, Switzerland.

UNISDR (2015), Sendai Framework for Disaster Risk Reduction 2015-2030, United Nations, Geneva, Switzerland. 